\title{
Spaceborne Underwater Imaging
}

\author{
Yoav Y. Schechner* \\ Dept. of Electrical Engineering \\ Technion - Israel Institute of Technology \\ Haifa 32000, Israel
}

\begin{abstract}
Shallow waters are very important for human and biological activity. Remote sensing of these areas is challenging, as it requires separation of ocean (or lake) bottom, water and atmospheric effects. In this paper we describe a concept and theory for spaceborne recovery of the underwater depth map, optical characteristics of the water and atmosphere, and the descattered ocean bottom. The sensing is based on multi-angular geometry and polarization. An orbiting platform captures a subspace of the Earth's light field, which is sensitive to the atmospheric and water characteristics. Consequently, it is possible to invert the image formation process using the acquired data. Recovery is simplified using recent findings about natural characteristics of deep water backscatter and surface transmissivity. It also exploits accumulated historical sounding data.
\end{abstract}

\section{Introduction}

Remote sensing of the ocean is important for oceanography, atmospheric sciences, and climatology. Thus, research satellite instruments ${ }^{1}$ are used for oceanic remote sensing, mainly in deep, open areas. However, shallow waters are important. Lakes, waterways, coral reefs and coastal waters possess a significant amount of biological activity [3], and are by far the most used and thus important for human activity [3]. Imaging in such regions is useful, thus, for biologists, geologists and archaeologists. Mapping there is important for boat safety, to avoid spots that are too shallow. It is also useful to oceanic engineers.

It is more difficult to analyze regions corresponding to shallow water. The open ocean is easier to analyze, since it is effectively dark in near-IR. This eases isolation and inversion of atmospheric effects [22, 26]. In shallow waters (termed Case $2[4,26]$ ), however, coastal constituents

* This work relates to Department of the Navy Grant N62909-10-1-4056 issued by the Office of Naval Research Global. The United States Government has a royalty-free license throughout the world in all copyrightable material contained herein.

${ }^{1}$ These include Polarization and Directionality of Earth's Reflectances (POLDER), MODerate resolution Imaging Spectroradiometer (MODIS), MEdium Resolution Imaging Spectrometer (MERIS), Sea-viewing Wide Field-of-view Sensor (SeaWiFs) and Multi-angle Imaging SpectroRadiometer (MISR)

\author{
David J. Diner, John V. Martonchik \\ Jet Propulsion Laboratory \\ California Institute of Technology \\ Pasadena, CA 91109
}

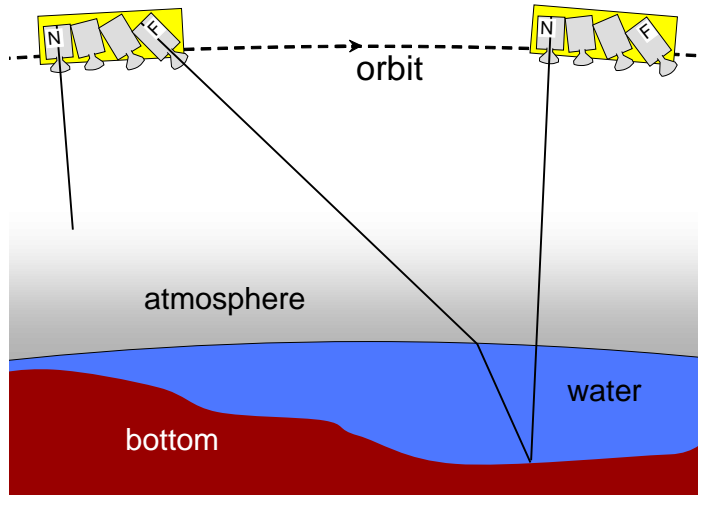

Figure 1. A satellite having multi-angular cameras. Camera $\mathrm{N}$ always looks at the nadir. Camera F looks obliquely forward, along the orbit track. In a short time, any surface point is sampled from multiple directions. Each view has systematically different levels of attenuation and path radiance by the water and atmosphere.

change the near-IR radiance, complicating IR-based recovery [26], and the sea bottom affects the radiance as well [4].

Remote depth estimation (bathymetry) of shallow areas has been impressively done using single-view hyperspectral data $[21,28,31]$. This approach assumes that ratios of attenuations at multiple wavelengths indicate depth. The estimated depth, however, changes with the albedo of the submerged object, since the approach does not invert the image formation model. Several parameters need to be tuned.

We propose an approach for remote underwater imaging, to recover: (1) the appearance of submerged objects, as if they are shallow; (2) the underwater depth map; (3) optical characteristics of the water, which imply the type and concentrations of hydrosols; (4) optical characteristics of the atmosphere, which imply the type and concentrations of aerosols. Goal (3) is important for studying and tracking the marine biological environment, specifically by recovering the concentration of chlorophyll $[3,26]$. It is also important for geological studies of ground deposits in water.

The theory in this paper shows that a multi-angular system (Fig. 1) which is polarization-sensitive appears to provide sufficient constraints, to essentially invert the image formation process and recover the important unknowns. We exploit the fact that shallow areas, being of human interest for ages, have had their depths sampled by boats. We 
also exploit scientific findings about natural characteristics of water polarization and deep water radiance. We believe this theory can suit a new generation of remote sensing instruments, having multiangular and polarimetric capabilities $[5,12]$, in addition to high spatial resolution.

\section{Background}

\subsection{Stokes and Mueller Formalism}

Let us view a partially linear polarized object. When rotating a polarizing filter in front of the camera, the measured intensity changes as a cosine of the filter orientation. The cosine has a $180^{\circ}$ period and three degrees of freedom, which depend on the scene: mean, amplitude and phase. Rather than using these parameters, the object state is conveniently parameterized [24] using an equivalent three-element Stokes vector. ${ }^{2}$ Let an object at very shallow water have Stokes vector $\mathbf{l}_{\mathrm{UW}}^{\mathrm{obj}}=\left[\begin{array}{lll}l_{\mathrm{UW}}^{\mathrm{obj}} & q_{\mathrm{UW}}^{\mathrm{obj}} & u_{\mathrm{UW}}^{\mathrm{obj}}\end{array}\right]^{\mathrm{T}}$, where $\mathrm{T}$ denotes transposition. Here, $l_{\mathrm{UW}}^{\mathrm{obj}}$ is the object radiance. The components $q_{\mathrm{UW}}^{\mathrm{obj}}$ and $u_{\mathrm{UW}}^{\mathrm{obj}}$ express polarization: their joint norm is equivalent to the above mentioned cosine amplitude, while their ratio is equivalent to the phase.

Incoherent superposition of beams is expressed by summing the respective Stokes vectors. Reflection, refraction and scattering instances, which are sensitive to the polarization components, are expressed by Mueller matrices, which operate on the Stokes vectors. Specifically, consider refraction through a flat water surface. Relative to the nadir, the submerged line of sight (LOS) is at an angle $\theta_{\mathrm{LOS}}^{\mathrm{w}}$. The submerged LOS is refracted to the airborne LOS, which is at angle $\theta_{\mathrm{LOS}}^{\mathrm{a}}$ relative to the zenith (See Fig. 2). For a horizontal water surface, Snell's law dictates that

$$
\sin \theta_{\mathrm{LOS}}^{\mathrm{a}}=n \sin \theta_{\mathrm{LOS}}^{\mathrm{w}},
$$

where $n$ is the optical refractive index of water (typically $n=1.34$ ). Across the water-air interface, transmission (refraction) of a ray associated with a Stokes vector is expressed $[18,24]$ by a Mueller matrix

$$
\mathbf{T}=\left[\begin{array}{ccc}
\left(t_{\|}+t_{\perp}\right) / 2 & \left(t_{\|}-t_{\perp}\right) / 2 & 0 \\
\left(t_{\|}-t_{\perp}\right) / 2 & \left(t_{\|}+t_{\perp}\right) / 2 & 0 \\
0 & 0 & \sqrt{t_{\|} t_{\perp}}
\end{array}\right]
$$

where

$$
\begin{aligned}
& t_{\|}=1-\left[\tan ^{2}\left(\theta_{\mathrm{LOS}}^{\mathrm{a}}-\theta_{\mathrm{LOS}}^{\mathrm{w}}\right)\right] /\left[\tan ^{2}\left(\theta_{\mathrm{LOS}}^{\mathrm{a}}+\theta_{\mathrm{LOS}}^{\mathrm{w}}\right)\right] \\
& t_{\perp}=1-\left[\sin ^{2}\left(\theta_{\mathrm{LOS}}^{\mathrm{a}}-\theta_{\mathrm{LOS}}^{\mathrm{w}}\right)\right] /\left[\sin ^{2}\left(\theta_{\mathrm{LOS}}^{\mathrm{a}}+\theta_{\mathrm{LOS}}^{\mathrm{w}}\right)\right]
\end{aligned}
$$

\subsection{Atmospheric Attenuation in Oblique View}

A camera flies above the atmosphere, and looks at the ocean. Had the camera pointed directly downwards, the radiance from the surface would have been filtered by the

\footnotetext{
${ }^{2}$ Stokes vectors have four elements, but we use a degenerate 3-element version of it, expressing only linear polarization. Similarly, we use degenerate $3 \times 3$ Mueller matrices, corresponding to linear polarization effects.
}

atmospheric transmissivity $\exp \left(-\tau^{\text {atm }}\right)$, where $\tau^{\text {atm }}$ is the optical depth of the atmosphere. However, the LOS in air is inclined at angle $\theta_{\mathrm{LOS}}^{\mathrm{a}}$. So, assuming a laterally uniform atmosphere [2, 22], its transmissivity along the LOS [22] is

$$
t^{\mathrm{atm}}=\exp \left(-\tau^{\mathrm{atm}} / \mu^{\mathrm{a}}\right), \quad \text { where } \mu^{\mathrm{a}} \equiv \cos \theta_{\mathrm{LOS}}^{\mathrm{a}}
$$

\subsection{Water Attenuation in Oblique View}

In water, the submerged LOS is at angle $\theta_{\mathrm{LOS}}^{\mathrm{w}}$ relative to the nadir (Fig. 2). Propagation of light from the object along the submerged LOS undergoes attenuation by the water, which is analogous to Eq. (5). Thus the water transmissivity along the LOS is

$$
t_{\mathrm{LOS}}^{\mathrm{w}}=\exp \left[-\beta^{\mathrm{w}} z(\mathbf{x}) / \mu^{\mathrm{w}}\right],
$$

where $z(\mathbf{x})$ is the underwater depth of the object at lateral location $\mathbf{x}=(x, y)$ on Earth. The depth is measured downwards from the ocean surface. Here $\mu^{\mathrm{w}}=\cos \theta_{\mathrm{LOS}}^{\mathrm{w}}$ and $\beta^{\mathrm{w}}$ is the attenuation coefficient of the water. Moreover, the object irradiance itself weakens with depth, since sunlight gets attenuated on the way down. Let the sun beam propagate at angle $\theta_{\text {sun }}^{\mathrm{w}}$ underwater, relative to the nadir. Similarly to Eq. (6), the water transmissivity along the lighting direction [11] is $t_{\mathrm{sun}}^{\mathrm{w}}=\exp \left[-\beta^{\mathrm{w}} z(\mathbf{x}) / \mu_{\mathrm{sun}}^{\mathrm{w}}\right]$, where $\mu_{\mathrm{sun}}^{\mathrm{w}}=\cos \theta_{\mathrm{sun}}^{\mathrm{w}}$. Overall, the object signal becomes dimmer with underwater depth, due to both effects: attenuation of irradiance and attenuation along the LOS. The combined effective transmissivity of the water, from the surface to the sea bottom and back to the surface [21] is

$$
t^{\mathrm{w}}(\mathbf{x})=t_{\mathrm{LOS}}^{\mathrm{w}} t_{\mathrm{sun}}^{\mathrm{w}}=\exp \left[-\beta^{\mathrm{w}} z(\mathbf{x})\left(\frac{1}{\mu_{\mathrm{sun}}^{\mathrm{w}}}+\frac{1}{\mu^{\mathrm{w}}}\right)\right]
$$

\section{Image Formation}

Let us describe a model for image formation when viewing objects through both the atmosphere and water. As shown in Fig. 3, the measured radiance $i(\mathbf{x})$ at the top of

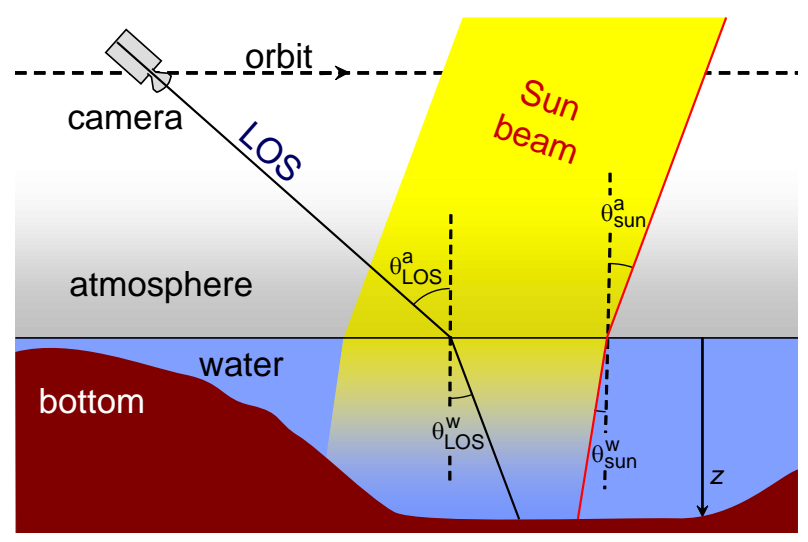

Figure 2. Sun rays irradiate the water surface at angle $\theta_{\text {sun }}^{\mathrm{a}}$ and refract at angle $\theta_{\mathrm{sun}}^{\mathrm{w}}$. Relative to the nadir, the camera's line of sight (LOS) is inclined at angle $\theta_{\mathrm{LOS}}^{\mathrm{a}}$ in air, i.e., at angle $\theta_{\mathrm{LOS}}^{\mathrm{w}}$ in water. The atmosphere and water are laterally homogeneous. 


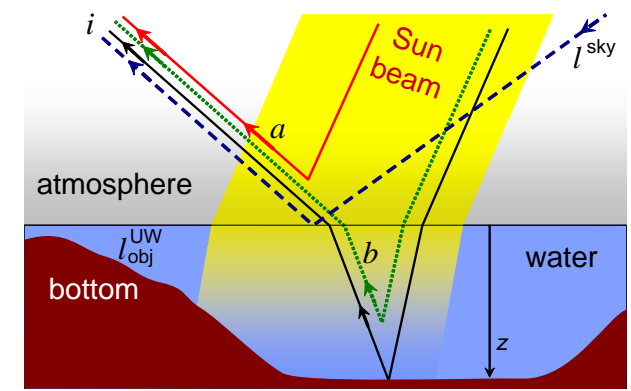

Figure 3. The measured intensity is composed of several components: airlight $a$, water backscatter $b$, and the object radiance, which is $l_{\mathrm{UW}}^{\mathrm{obj}}$ if the object is very shallow. The camera points away from sun-glint, and senses a reflection of the sky radiance $l^{\text {sky }}$. The bottom is at underwater depth $z$.

the atmosphere (TOA) is affected by several components:

- A submerged object. Had it been very close to the surface [36], its radiance would have been $l_{\mathrm{UW}}^{\mathrm{obj}}$. When the object is submerged deeper, its radiance is attenuated by the water. Propagating upwards along the LOS, the object radiance is further reduced by the transmissivity of the water surface $t_{\text {surface }}$ and the atmospheric transmissivity (5).

- Scattering in the water creates water path radiance along the LOS. This path radiance is sometimes referred to as backscatter [29], and is denoted here by $b$. On its propagation upwards along the LOS, the backscatter is reduced by the transmissivity of the water surface and the atmosphere.

- A portion of the sky having effective radiance $l^{\text {sky }}$ is reflected by the water surface, ${ }^{3}$ whose reflectivity is $r_{\text {surface }}$. Also this component suffers from atmospheric attenuation.

- Light which is unrelated to the object at a specific pixel irradiates the atmosphere. A direct irradiance source is sunlight. Indirect sources include multiple scattering in the atmosphere (skylight) and diffuse lighting from water and land surrounding the field of view (FOV). Part of that light is scattered towards camera along the LOS, creating an atmospheric path radiance, referred to as airlight [8, 27, 30, 32], and denoted here by $a$.

Overall, at the TOA,

$i(\mathbf{x})=$

$\left\{\left[l_{\mathrm{UW}}^{\mathrm{obj}}(\mathbf{x}) t^{\mathrm{w}}(\mathbf{x})+b(\mathbf{x})\right] t_{\text {surface }}+l^{\text {sky }} r_{\text {surface }}\right\} t^{\text {atm }}+a$.

According to Ref. [11, 21],

$$
b(\mathbf{x})=b_{\infty}\left[1-t^{\mathrm{w}}(\mathbf{x})\right],
$$

regardless of the slope of the LOS and the attenuation of irradiance with depth. Here $b_{\infty}$ is the asymptotic value of $b$ corresponding to an infinitely deep sea bottom.

To generalize (8) to polarization fields, scalar radiance is replaced by a Stokes vector. The respective vectors of

\footnotetext{
${ }^{3}$ Scattering by whitecap waves is another component. Our analysis focuses on weather conditions which do not create significant whitecaps.
}

airlight, the sky patch reflected by the water and backscatter are $\mathbf{a}, \mathbf{l}^{\text {sky }}$ and $\mathbf{b}=\left[b q^{\mathrm{b}} u^{\mathrm{b}}\right]^{\mathrm{T}}$. According to [11], similarly to Eq. (8), $\mathbf{b}(\mathbf{x}) \approx \mathbf{b}_{\infty}\left[1-t^{\mathrm{w}}(\mathbf{x})\right]$. Here $\mathbf{b}_{\infty}$ is the polarization state of backscatter along an LOS submerged to an effectively infinite depth. Transmissivities of bulk water and bulk atmosphere have no polarization sensitivity, thus overall the Stokes vector measured at the camera is

$$
\mathbf{i}(\mathbf{x})=t^{\mathrm{atm}}\left\{\mathbf{T}\left[t^{\mathrm{w}}(\mathbf{x}) \mathbf{l}_{\mathrm{UW}}^{\mathrm{obj}}(\mathbf{x})+\mathbf{b}(\mathbf{x})\right]+\mathbf{R} \mathbf{l}^{\mathrm{sky}}\right\}+\mathbf{a} .
$$

Here $\mathbf{R}$ and $\mathbf{T}$ are Mueller matrices of water surface (10)
flection and transmission (refraction), respectively, while $\mathbf{R}[1,1]=r_{\text {surface }}$ and $\mathbf{T}[1,1]=t_{\text {surface }}$. Both matrices are governed by the Fresnel equations [24], and depend on the angle between the LOS and the water surface normal. At remote sensing scales, the water is horizontal, but somewhat rough due to water waves. The matrix $\mathbf{R}$ is discussed in Ref. [18], particularly in the context of wavy water. The vector $\mathbf{R l}^{\text {sky }}$ is sensitive to the wave conditions. However, as we shall soon see, we do not need $\mathbf{R} \mathbf{l}^{\text {sky }}$. Thus, we skip its details. The matrix $\mathbf{T}$ is detailed in Eq. (2) for flat water, but what happens in a wavy sea? Fortunately, (2) holds even in wavy conditions, as we discuss in Sec. 4.

\section{Prior Knowledge}

In addition to the model, we employ knowledge which has been accumulated by marine researchers and users.

1. In some lateral coordinates $\mathbf{x}^{\mathrm{s}}$, good assessment [21] of the underwater depth $z$ exists. It stems from sample depth sounding of shallow waters, which has been performed for many years. The discrete set of points for which $z\left(\mathbf{x}^{\mathrm{s}}\right)$ is known prior to our recovery is termed here soundings. Most of the FOV has unknown $z$.

2. Blur due to refraction by a wavy water surface is negligible, at the relevant scales we work in. This fact is used also by existing methods of multispectral bathymetry [21, 31]. Hence, we do not attempt to measure the wave structure $[15,16]$.

3. Around the nadir, $b_{\infty}$ is at a rather flat minimum, as revealed by Refs. $[2,25,34,35]$. Hence, we model it as

$$
b_{\infty}\left(\mu^{\mathrm{w}}\right) \approx b_{\infty}(\mathrm{N})+\alpha\left(1-\mu^{\mathrm{w}}\right),
$$

where $b_{\infty}(\mathrm{N})$ is the value of $b_{\infty}$ at the nadir $\left(\mu^{\mathrm{w}}=1\right)$, and $\alpha \ll b_{\infty}(\mathrm{N})$ is a global constant.

4. Refraction induces a predictable polarizance expressed by $\mathbf{T}$. It can be compensated. Deviations due to water surface waves have a negligible effect. As shown in Fig. 4, $\mathbf{T}$ is almost invariant to changes in the angles of incidence, for a fairly broad range around the surface normal. Thus, when waves change the angle of incidence, they hardly change the transmittance. Second, a pixel in a satellite camera does not sense individual wave patches, 
but averages a wide area, integrating over surface slopes. Ref. [10] derives the strong insensitivity of the surface transmissivity to the wind conditions. As a result, flat water (no wind) transmittance suffices for our needs.

\section{Assumptions}

For a simple inversion, we make several assumptions.

I . Across the FOV, the atmosphere and the water are laterally homogeneous: their parameters are constant. This is commonly assumed in remote sensing [2, 22].

II. One sounding is in very deep water [21], where $z \rightarrow \infty$, and thus $t^{\mathrm{w}} \rightarrow 0$. There, the measured Stokes vector at the TOA is

$$
\mathbf{i}_{\text {deep }}=t^{\text {atm }}\left[\mathbf{R} l^{\text {sky }}+\mathbf{T} \mathbf{b}_{\infty}\right]+\mathbf{a} .
$$

III. Blur due to scattering is negligible. Multiple scattering can exist, but overall, blurring between nearby small targets is neglected. The multiply scattered (blurred) light from small targets is assumed to get attenuated with distance similarly to singly scattered light, and the error in this assumption is assumed to be small, relative to the total signal. Neglecting blur is successful in a range of outdoors works [14, 19, 20, 27, 29, 30, 32] in computer vision. It is more valid in the current work, since usually the optical thickness is smaller in a view from above, than in open country horizontal views. While the satellite is hundreds of kilometers high, it is mainly the lowest few kilometers in which dense scatterers reside. Hence, blur is also neglected in multispectral bathymetric estimation [21, 28]. Multiple scattering from diffuse, large sources (sky light, or light radiated by the whole sea to the horizon) can be significant. However, these components also add-up in pixels corresponding to deep water. As we shall see, we use the deep water radiance to cancel global, diffuse components. Thus, such components are countered by our method.

IV . The submerged object at a sounding reflects unpolarized light [29, 30]. Specularity underwater is weaker [29], since refractive index mismatches are smaller there. Some objects in the FOV may be partially polarized, though.

$\mathrm{V}$. The object is effectively Lambertian when submerged. This is supported by the reduced specularity just described.

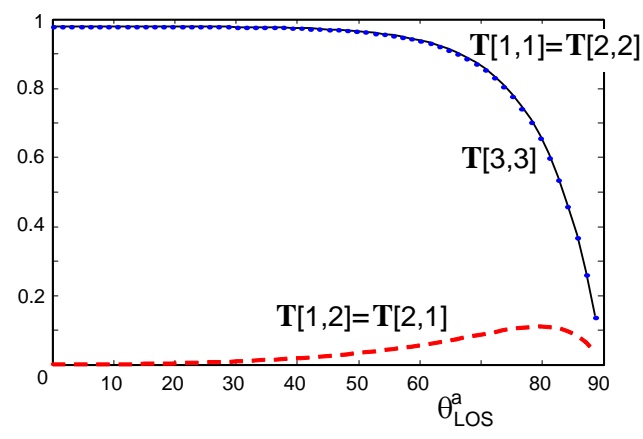

Figure 4. The non-zero elements of $\mathbf{T}$.
Moreover, Ref. [36] shows that empirically, submerged coastal materials are significantly more Lambertian than in air, particularly when illuminated and viewed from roughly above, as in our geometry. It is sufficient for this assumption to hold within our range of $\theta_{\mathrm{LOS}}^{\mathrm{w}}$, which is significantly smaller than the range of $\theta_{\mathrm{LOS}}^{\mathrm{a}}$, due to refraction.

\section{Descattering and Depth Map Recovery}

Recovering any of the unknowns in this paper is essentially a parametric fitting problem. Thus, we will show how the data yields constraints for a fit. Section 7 describes how the spatially invariant parameters $\left\{\beta^{\mathrm{w}}, \tau^{\text {atm }}, \alpha\right\}$ can be estimated, based on soundings data and priors. Thus, in the current section, assume that the respective estimates $\left\{\hat{\beta}^{\mathrm{w}}, \hat{\tau}^{\mathrm{atm}}, \hat{\alpha}\right\}$ exist. Based on them, multi angular acquisition by the satellite is exploited here to estimate the unknown spatially varying $z(\mathbf{x})$ and $l_{\mathrm{UW}}^{\mathrm{obj}}(\mathbf{x})$. The submerged objects can be arbitrarily polarized, since we make no use of polarization here.

At a pixel viewing deep water, the radiance of Eq. (12) is $i_{\text {deep }}=t^{\text {atm }}\left(r_{\text {surface }} l^{\text {sky }}+t_{\text {surface }} b_{\infty}\right)+a$, where all the variables depend on $\theta_{\mathrm{LOS}}^{\mathrm{a}}$. Following Eqs. $(8,9)$, define

$$
i_{\text {undeep }}\left(\mathbf{x}, \mu^{\mathrm{a}}\right) \equiv i\left(\mathbf{x}, \mu^{\mathrm{a}}\right)-i_{\text {deep }}\left(\mu^{\mathrm{a}}\right) \text {. }
$$

Then, Eqs. $(8,9,13)$ yield

$$
i_{\text {undeep }}\left(\mathbf{x}, \mu^{\mathrm{a}}\right)=t_{\text {surface }}\left[l_{\mathrm{UW}}^{\mathrm{obj}}(\mathbf{x})-b_{\infty}\right] t^{\text {watm }}\left(\mathbf{x}, \mu^{\mathrm{a}}\right) \text {, }
$$

where

$$
t^{\mathrm{watm}}=t^{\mathrm{w}}(\mathbf{x}) t^{\mathrm{atm}} .
$$

The simple calculation in Eq. (13) thus eliminates the unknown atmospheric path-radiance and surface reflection, in each view setting $\mu^{\mathrm{a}}$, as seen in Eq. (14).

Since $t_{\text {surface }}$ is practically unaffected [10] by the waves, it can be derived using Eq. (2) and be easily inverted. For most angles $\theta_{\mathrm{LOS}}^{\mathrm{a}}$, the inversion is very stable since $t_{\text {surface }} \approx 1$, yielding

$$
i_{\text {unsurf }}\left(\mathbf{x}, \mu^{\mathrm{a}}\right)=i_{\text {undeep }}\left(\mathbf{x}, \mu^{\mathrm{a}}\right) /\left[t_{\text {surface }}\left(\mu^{\mathrm{a}}\right)\right] .
$$

Based on $\hat{\tau}^{\mathrm{atm}}$, define

$$
i_{\text {atm }}^{\text {no }}\left(\mathbf{x}, \mu^{\mathrm{a}}\right)=i_{\text {unsurf }}\left(\mathbf{x}, \mu^{\mathrm{a}}\right) \exp \left(\hat{\tau}^{\mathrm{atm}} / \mu^{\mathrm{a}}\right) \text {. }
$$

Based on Eqs. $(14,16,17), i_{\mathrm{atm}}^{\mathrm{no}}$ is compensated for both the surface transmissivity and the atmospheric effects (airlight and attenuation) along the LOS. Define

$$
l_{\mathrm{N}}^{\mathrm{obj}}(\mathbf{x})=l_{\mathrm{UW}}^{\mathrm{obj}}(\mathbf{x})-b_{\infty}(\mathrm{N})
$$

According to Eqs. $(7,11,14,17,18)$,

$i_{\mathrm{atm}}^{\mathrm{no}}\left(\mathbf{x}, \mu^{\mathrm{a}}\right)=\left[l_{\mathrm{N}}^{\mathrm{obj}}(\mathbf{x})-\alpha\left(1-\mu^{\mathrm{w}}\right)\right] e^{-\left(\frac{1}{\mu_{\mathrm{sun}}^{\mathrm{w}}}+\frac{1}{\mu^{\mathrm{w}}}\right) \beta^{\mathrm{w}} z(\mathbf{x})}$.

The orbiting satellite acquires images at a set of known $M>3$ elevation angles $\theta_{\mathrm{LOS}}^{\mathrm{a}}$, i.e., known values of $\left\{\mu_{m}^{\mathrm{a}}\right\}_{m=1}^{M}$. Correspondingly, the set $\left\{\mu_{m}^{\mathrm{w}}\right\}_{m=1}^{M}$ 
is known as well, using a cosine version of Snell's law, $\mu^{\mathrm{w}}=\frac{1}{n} \sqrt{n^{2}-1+\left(\mu^{\mathrm{a}}\right)^{2}}$. The sun state $\mu_{\text {sun }}^{\mathrm{w}}$ is known as well. Given the spatially invariant $\left\{\beta^{\mathrm{w}}, \alpha\right\}$, Eq. (19), has only two unknowns per pixel: the biased scatter-free $l_{\mathrm{N}}^{\mathrm{obj}}(\mathbf{x})$, and the depth $z(\mathbf{x})$. Yet, per pixel there is a set of $M$ empirically derived values $\left\{i_{\text {atm }}^{\text {no }}\left(\mathbf{x}, \mu_{m}^{\mathrm{a}}\right)\right\}_{m=1}^{M}$, which independently constrain the two unknowns through the nonlinear relation (19). Thus, estimating $z(\mathbf{x})$ and $l_{\mathrm{N}}^{\mathrm{obj}}(\mathbf{x})$ is over-constrained $\forall \mathbf{x}$, thanks to multiview sensing.

In practice, the vector $\left[z, l_{\mathrm{N}}^{\mathrm{obj}}\right]_{\mathbf{x}}$ can be searched to fit Eq. (19) in multiple view angles simultaneously. The search domain is finite: $z \in(0,50 \mathrm{~m})$, as 50 meters are already in the "very deep" domain, while the object reflectivity domain is not different than that of dry objects.

The parameter $b_{\infty}(\mathrm{N})$ is not recovered in this work. Hence, Eq. (18) is not inverted to completely recover $l_{\mathrm{UW}}^{\mathrm{obj}}(\mathbf{x})$. Thus, the object radiance is recovered up to a global bias. The bias is small, since usually $b_{\infty}(\mathrm{N})$ is significantly smaller than $l_{\mathrm{UW}}^{\mathrm{obj}}(\mathbf{x})$.

\section{Simulation}

As an example, we performed a simulation. The simulation inputs are detailed below. Some parameters are inspired by the MISR instrument, which has been acquiring global multi-angle imagery of Earth aboard NASA's Terra satellite since 2000. It has nine pushbroom cameras arrayed at nine along-track view angles.

Optical bands: Red=671.7 nm, Green=557.5 nm, Blue $=446.4 \mathrm{~nm}$, as the visible MISR wavelength channels.

Atmosphere: Spherical particles with a bimodal size distribution, where each mode is a log-normal distribution. In mode 1 , the median radius is 0.03 micron, and the standard deviation (STD) of the natural log of radius is 0.501 . In mode 2 , the median radius is 1 micron, and the STD of the natural $\log$ of radius is 0.642 . In both modes, the real refractive index is 1.45 and the imaginary refractive index is 0 . In the green band, modes 1 and 2 account, respectively, for $70 \%$ and $30 \%$ of the optical depth. The tropospheric aerosol base altitude, top altitude and scale height are $0 \mathrm{~km}, 10 \mathrm{~km}$ and $2 \mathrm{~km}$, respectively. Including Rayleigh scattering, the total atmospheric optical depths are $\left[\tau_{\text {Red }}^{\text {atm }}, \tau_{\text {Green }}^{\text {atm }}, \tau_{\text {Blue }}^{\text {atm }}\right]=[0.05716,0.1122,0.262]$.

Camera zenith angles [degrees]: Similar to MISR, $\theta_{\mathrm{LOS}}^{\mathrm{a}} \in\{70.4,60.3,45.9,26.5,3.1,26,45.5,60,70.3\}$.

Solar zenith angle: $65^{\circ}$.

Ocean water: Albedo and surface reflectance distributions are those used by MISR's retrieval products [22, 23], for 2 meter/sec wind-speed. The attenuation coefficients are $\left[\beta_{\text {Red }}^{\mathrm{w}}, \beta_{\text {Green }}^{\mathrm{w}}, \beta_{\text {Blue }}^{\mathrm{w}}\right]=[1 / 5,1 / 20,1 / 10]$ meter $^{-1}$.

Object: The reflectance texture is shown in Fig. 5. This image was taken since it has average color balance (in the respective wavelength bands) as a quartz beach sample from the Gulf of Mexico (Sample 110 in [1]). The colors in the image were slightly adapted to fit the beach sand spectral data. The absolute reflectance was reduced by $45 \%$, relative to the values in [1], to emulate darkening of submerged sand [36]. The depth map is shown in Fig. 5.

Sensor: Similarly to MISR, pixel full well is $10^{6} \mathrm{e}^{-}$. It corresponds to the brightest readout. Photon noise dominates in such bright images, and it was thus incorporated.

To analyze the images, we first created a look-up table (LUT) of discrete $\left[z, l_{\mathrm{N}}^{\mathrm{obj}}\right]$ values. Then, for each pixel, the LUT was searched for the best fitting vector, which is denoted $\left[\hat{z}, \hat{l}_{\mathrm{N}}^{\mathrm{obj}}\right]$. The fit was done, per pixel, by minimizing the sum of absolute differences, along all $\theta_{\mathrm{LOS}}^{\mathrm{a}}$, between the two sides of Eq. (19). The results have some outliers, so a simple spatial median filter was applied as post precessing. It is illustrative to look at the results of the blue channel, for which, in this simulation $\beta^{\mathrm{w}}=1 / 10$ meter $^{-1}$, i.e., the median attenuation among the color channels.

According to the scatter plot, the estimated depths follow the correct depth, until nearly 20 meters. This is twice the attenuation length of the water. But, light passes back and forth from the water surface, and the sun illuminates from a rather shallow slope. So, light is being attenuated for significantly more than twice the attenuation length. Thus, around 20 meters, the recovery breaks down, and large errors occur. This is also seen in the estimated radiance $\hat{l}_{\mathrm{N}}^{\mathrm{obj}}$ : there is nice recovery in the northern part of the image, but the southern portion, corresponding to 20 meters and deeper, demonstrates quick deterioration. There, the lack of object signal is interpreted as a dark object at a random depth. Consequently, the radiance scatter plot has two clusters. One cluster nicely fits the true radiance $l_{\mathrm{UW}}^{\mathrm{obj}}$ (along the main slope in the plot). The other cluster is around $\hat{l}_{\mathrm{N}}^{\mathrm{obj}}=0$, corresponding the erroneously dark region in the recovery.

Recovery of somewhat deep regions, which fails here, is not lost. As shown in Ref. [17], regularized solutions which are adaptive to depth can suppress noisy results in deep regions. This is contrary to the recovery attempted here, which is essentially pointwise. However, recovery of deep regions leads to fast loss of spatial resolution, due to the low signal-to-noise ratio there [33], despite having no spatial blur in the optical model. Since photon noise is dominant, it is best to minimize, during acquisition, the sky reflection and airlight. Thus, the method should avoid images containing sun-glint. This is often achievable: thanks to the multi-angular setup, if some cameras suffer from sun glint, then others may not point in the glint direction.

The radiance scatter plot shows that some pixels have a negative value for $\hat{l}_{\mathrm{N}}^{\mathrm{obj}}$. This is fine, since according to Eq. (18), $l_{\mathrm{N}}^{\mathrm{obj}}<l_{\mathrm{UW}}^{\mathrm{obj}}$ due to a negative bias. However, the plot shows that the systematic bias is not large, relative to errors stemming from the random noise. Hence, $l_{\mathrm{N}}^{\mathrm{obj}}$ can 

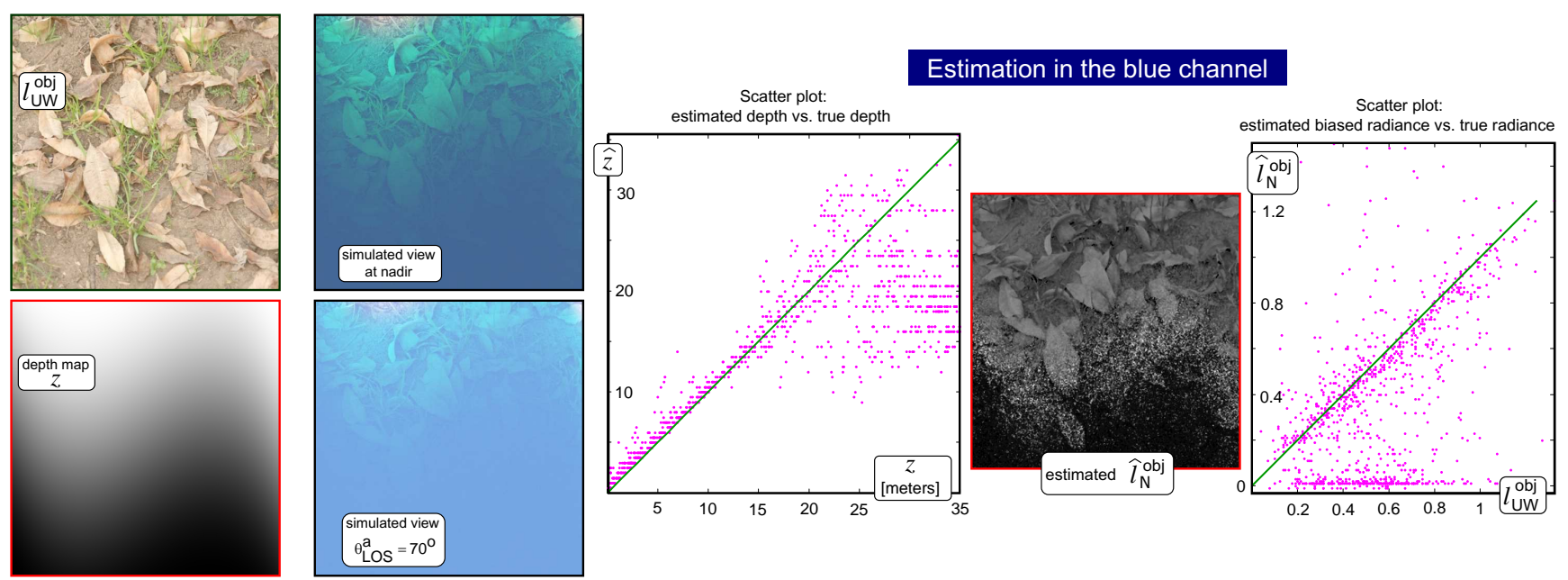

Figure 5. Left to right: input to image rendering; two simulated frames (out of nine views), which are input the to recovery step; recovery results. The displayed images are gamma-corrected for clarity. Shallowest and deepest points are at $z=0$ and $z=35$ meters, and are in the brightest a darkest pixels, respectively, in the depth map. Scatter plots are based on 1000 random points in the FOV.

often be a good approximation to $l_{\mathrm{UW}}^{\mathrm{obj}}$.

\section{Estimation of Scene Parameters \\ 7.1. The Atmospheric Optical Depth}

There are algorithms for atmospheric correction of satellite images over ocean [2, 4, 22], including estimation of the atmospheric optical depth $\tau^{\text {atm }}$. They are rigorous, and account for multiple scattering and types of aerosols, hence may work in our case. On the other hand, as discussed in Sec. 1, they are challenged by shallow water scenarios. We describe here a way to approach the problem.

It is important to estimate the water attenuation coefficient, $\beta^{\mathrm{w}}$. After $\beta^{\mathrm{w}}$ is recovered, $\tau^{\text {atm }}$ can be estimated. However, to ease the flow of the paper, we first describe the estimation $\tau^{\mathrm{atm}}$, assuming, for the time, that $\beta^{\mathrm{w}}$ is known. To calibrate the parameters, we use the data in pixels $\mathbf{x}^{\mathrm{s}}$ corresponding to soundings. At soundings, the depth $z\left(\mathbf{x}^{\mathrm{s}}\right)$ is known, and so is the water attenuation $t^{\mathrm{w}}\left(\mathrm{x}^{\mathrm{s}}\right)$, via Eq. (7).

Equation (16) is derived directly from the raw data, without prior parameter estimation. Compensating for water attenuation at the soundings and using $(5,11)$ yields

$i_{\text {dry }}\left(\mathbf{x}^{\mathrm{s}}\right)=\frac{i_{\text {unsurf }}\left(\mathbf{x}^{\mathrm{s}}\right)}{t^{\mathrm{w}}\left(\mathbf{x}^{\mathrm{s}}\right)}=\left[l_{\mathrm{N}}^{\mathrm{obj}}\left(\mathbf{x}^{\mathrm{s}}\right)-\alpha\left(1-\mu^{\mathrm{w}}\right)\right] e^{-\tau^{\mathrm{atm}} / \mu^{\mathrm{a}}}$.

Eq. (20) has three unknowns: the local $l_{\mathrm{N}}^{\mathrm{obj}}\left(\mathbf{x}^{\mathrm{s}}\right)$ at a sounding, and the global (and of interest) $\left\{\tau^{\mathrm{atm}}, \alpha\right\}$. On the other hand, the cameras acquire images at a set of known $M>3$ elevation angle settings $\left\{\mu_{m}^{\mathrm{a}}\right\}_{m=1}^{M}$. Thus, the parameters can be recovered by fitting the data at $\mathbf{x}^{\mathrm{s}}$ to Eq. (20).

We now describe a simplified process. We may write

$$
l_{\mathrm{N}}^{\mathrm{obj}}\left(\mathbf{x}^{\mathrm{s}}\right)-\alpha\left(1-\mu^{\mathrm{w}}\right) \approx l_{\mathrm{N}}^{\mathrm{obj}}\left(\mathbf{x}^{\mathrm{s}}\right)\left[1+\left(1-\mu^{\mathrm{a}}\right) \omega\left(\mathbf{x}^{\mathrm{s}}\right)\right] .
$$

Here $\omega\left(\mathbf{x}^{\mathbf{s}}\right)$ is a small unknown. It is angularly-constant, but changes from pixel to pixel. At the nadir, $\mu^{\mathrm{a}}=1$, leading to an exremum value $l_{\mathrm{N}}^{\mathrm{obj}}\left(\mathbf{x}^{\mathrm{s}}\right)$ at the right hand side of Eq. (21). Off nadir, as $\theta_{\mathrm{LOS}}^{\mathrm{a}}$ increases, both sides of Eq. (21) slowly change quadratically (at small angles). From Eqs. (20,21),

$$
\begin{aligned}
& i_{\text {dry }}\left(\mathbf{x}^{\mathrm{s}}, \mu^{\mathrm{a}}\right) \approx l_{\mathrm{N}}^{\mathrm{obj}}\left(\mathbf{x}^{\mathrm{s}}\right)\left[1+\left(1-\mu^{\mathrm{a}}\right) \omega\left(\mathbf{x}^{\mathrm{s}}\right)\right] e^{-\tau^{\mathrm{atm}} / \mu^{\mathrm{a}}} . \\
& \text { Define } \tilde{i}_{\mathrm{dry}}=\log i_{\mathrm{dry}} \text { and } \tilde{l}_{\mathrm{N}}^{\text {obj }}=\log l_{\mathrm{N}}^{\text {obj }} \text {. Then } \\
& \tilde{i}_{\mathrm{dry}}\left(\mathbf{x}^{\mathrm{s}}, \mu^{\mathrm{a}}\right) \approx \tilde{l}_{\mathrm{N}}^{\text {obj }}\left(\mathbf{x}^{\mathrm{s}}\right)+\left(1-\mu^{\mathrm{a}}\right) \omega\left(\mathbf{x}^{\mathrm{s}}\right)-\tau^{\mathrm{atm}} / \mu^{\mathrm{a}},
\end{aligned}
$$

where we use the relation $\log \left[1+\left(1-\mu^{\mathrm{a}}\right) \omega\right] \approx\left(1-\mu^{\mathrm{a}}\right) \omega$, when $\left(1-\mu^{\mathrm{a}}\right) \omega$ is small. Thus, for each $\mathbf{x}^{\mathrm{s}}$, there is a set of equations (23), which can be written as

$$
\left[\begin{array}{ccc}
1 & \left(1-\mu_{1}^{\mathrm{a}}\right) & \frac{-1}{\mu_{1}^{\mathrm{a}}} \\
1 & \left(1-\mu_{2}^{\mathrm{a}}\right) & \frac{-1}{\mu_{2}^{\mathrm{a}}} \\
\vdots & \vdots & \vdots \\
1 & \left(1-\mu_{M}^{\mathrm{a}}\right) & \frac{-1}{\mu_{M}^{\mathrm{a}}}
\end{array}\right]\left[\begin{array}{l}
\tilde{l}_{\mathrm{N}}^{\mathrm{obj}}\left(\mathbf{x}^{\mathrm{s}}\right) \\
\omega\left(\mathbf{x}^{\mathrm{s}}\right) \\
\tau^{\mathrm{atm}}
\end{array}\right]=\left[\begin{array}{l}
\tilde{i}_{\mathrm{dry}}\left(\mu_{1}^{\mathrm{a}}\right) \\
\tilde{i}_{\mathrm{dry}}\left(\mu_{2}^{\mathrm{a}}\right) \\
\vdots \\
\tilde{i}_{\mathrm{dry}}\left(\mu_{M}^{\mathrm{a}}\right)
\end{array}\right]
$$

This set of equations solved, to estimate the unknowns $\tilde{l}_{\mathrm{N}}^{\mathrm{obj}}\left(\mathbf{x}^{\mathrm{s}}\right), \omega\left(\mathbf{x}^{\mathrm{s}}\right)$, and most importantly in this section, $\tau^{\mathrm{atm}}$. Once the estimation of $\tau^{\text {atm }}$ (and $l_{\mathrm{N}}^{\text {obj }}\left(\mathbf{x}^{\mathrm{s}}\right)$ ) is done, fitting Eq. (20) to recover $\alpha$ becomes simple.

\subsection{The Water Attenuation Coefficient}

Equation (16) is obtained directly from the raw data, without prior parameter estimation. Spatial variations in Eq. (16) are affected by both the object radiance $l_{\mathrm{UW}}^{\mathrm{obj}}(\mathbf{x})$ and the water transmissivity $t^{\mathrm{w}}(\mathbf{x})$. We need to decouple these functions, in order to recover the water transmissivity. This is the place where polarization is useful. We use polarization at pixels $\mathbf{x}^{\mathrm{s}}$ corresponding to soundings.

Generalizing Eq. (13) to polarization, define the empirically derived field $\mathbf{i}_{\text {undeep }}\left(\mathbf{x}^{\mathrm{s}}\right) \equiv \mathbf{i}\left(\mathbf{x}^{\mathrm{s}}\right)-\mathbf{i}_{\text {deep }}$, where (following Eqs. 10,15,12) 


$$
\mathbf{i}_{\text {undeep }}\left(\mathbf{x}^{\mathrm{s}}\right)=\mathbf{T}\left[\mathbf{l}_{\mathrm{UW}}^{\mathrm{obj}}\left(\mathbf{x}^{\mathrm{s}}\right)-\mathbf{b}_{\infty}\right] t^{\mathrm{watm}}\left(\mathbf{x}^{\mathrm{s}}\right) .
$$

From assumption IV, $\mathbf{l}_{\mathrm{UW}}^{\mathrm{obj}}$ is unpolarized. Thus,

$$
\mathrm{l}_{\mathrm{UW}}^{\mathrm{obj}}\left(\mathbf{x}^{\mathrm{s}}\right)=\left[\begin{array}{lll}
l_{\mathrm{UW}}^{\mathrm{obj}}\left(\mathbf{x}^{\mathrm{s}}\right) & 0 & 0
\end{array}\right]^{\mathrm{T}} .
$$

Using Eq. (26) in Eq. (25) yields

$\mathbf{i}_{\text {undeep }}\left(\mathbf{x}^{\mathrm{s}}\right)=\mathbf{T}\left\{\left[\begin{array}{c}l_{\mathrm{UW}}^{\mathrm{obj}}\left(\mathbf{x}^{\mathrm{s}}\right) \\ 0 \\ 0\end{array}\right]-\left[\begin{array}{c}b_{\infty} \\ u_{\infty}^{\mathrm{b}} \\ q_{\infty}^{\mathrm{b}}\end{array}\right]\right\} t^{\mathrm{watm}}\left(\mathbf{x}^{\mathrm{s}}\right)$.

The backscatter polarization components $u_{\infty}^{\mathrm{b}}$ and $q_{\infty}^{\mathrm{b}}$ are spatially invariant and are not mixed with polarized components of an object. Furthermore, $\mathbf{T}$ is spatially invariant as well. Hence, focusing on the polarized components in Eq. (27) should assist in the desired decoupling.

Following prior $4, \mathbf{T}$ is predictable and stable even in unknown wavy conditions, and is given by the flat water expression Eq. (2). As shown in Ref. [18], for most angles of interest, $\mathbf{T}$ is strongly diagonal - nearly the identity matrix. Hence, its inversion is simple and stable. The surface polarizance is thus compensated by

$$
\left[\begin{array}{c}
i_{\text {unsurf }}\left(\mathbf{x}^{\mathrm{s}}\right) \\
u_{\text {unsurf }}\left(\mathbf{x}^{\mathrm{s}}\right) \\
q_{\text {unsurf }}\left(\mathbf{x}^{\mathrm{s}}\right)
\end{array}\right]=\mathbf{i}_{\text {unsurf }}\left(\mathbf{x}^{\mathrm{s}}\right)=\hat{\mathbf{T}}^{-1} \mathbf{i}_{\text {undeep }}\left(\mathbf{x}^{\mathrm{s}}\right) .
$$

The polarized vector component of $\mathbf{i}_{\text {unsurf }}\left(\mathbf{x}^{\mathrm{s}}\right)$ has magnitude

$$
i^{\mathrm{pol}}\left(\mathbf{x}^{\mathrm{s}}\right)=\sqrt{u_{\text {unsurf }}^{2}\left(\mathbf{x}^{\mathrm{s}}\right)+q_{\text {unsurf }}^{2}\left(\mathbf{x}^{\mathrm{s}}\right)} .
$$

From Eqs. $(15,27,28), u_{\text {unsurf }}\left(\mathbf{x}^{\mathrm{s}}\right)=u_{\infty}^{\mathrm{b}} t^{\mathrm{atm}} t^{\mathrm{w}}\left(\mathbf{x}^{\mathrm{s}}\right)$ and $q_{\text {unsurf }}\left(\mathbf{x}^{\mathrm{s}}\right)=q_{\infty}^{\mathrm{b}} t^{\mathrm{atm}} t^{\mathrm{w}}\left(\mathbf{x}^{\mathrm{s}}\right)$. Hence,

$$
i^{\mathrm{pol}}\left(\mathbf{x}^{\mathrm{s}}\right)=\sqrt{\left(u_{\infty}^{\mathrm{b}}\right)^{2}+\left(q_{\infty}^{\mathrm{b}}\right)^{2}} t^{\mathrm{atm}} t^{\mathrm{w}}\left(\mathbf{x}^{\mathrm{s}}\right) .
$$

Now, the spatial variations of the empirically measured $i^{\mathrm{pol}}(\mathbf{x})$ depend only on the spatial variations of $t^{\mathrm{w}}(\mathbf{x})$.

Define $\tilde{i}^{\mathrm{pol}}\left(\mathbf{x}^{\mathrm{s}}\right)=\log i^{\mathrm{pol}}\left(\mathbf{x}^{\mathrm{s}}\right)$. Incorporating Eq. (7) in Eq. (30), and taking the log,

$$
\tilde{i}^{\mathrm{pol}}\left(\mathbf{x}^{\mathrm{s}}\right)=C-\beta^{\mathrm{w}} z\left(\mathbf{x}^{\mathrm{s}}\right)\left[\left(1 / \mu_{\mathrm{sun}}^{\mathrm{w}}\right)+\left(1 / \mu^{\mathrm{w}}\right)\right],
$$

where

$$
C=\log \left[t^{\mathrm{atm}} \sqrt{\left(u_{\infty}^{\mathrm{b}}\right)^{2}+\left(q_{\infty}^{\mathrm{b}}\right)^{2}}\right]
$$

is spatially invariant. The view angle $\theta_{\mathrm{LOS}}^{\mathrm{a}}$ of any orbiting camera is known, as well as the fixed $\theta_{\text {sun }}^{\text {a }}$. Thus, $\left\{\mu^{\mathrm{w}}, \mu_{\text {sun }}^{\mathrm{w}}\right\}$ are known. Furthermore, according to prior 1 $z\left(\mathbf{x}^{\mathrm{s}}\right)$ is known (soundings). Hence,

$$
\tilde{z}\left(\mathbf{x}^{\mathrm{s}}\right) \equiv z\left(\mathbf{x}^{\mathrm{s}}\right)\left[\left(1 / \mu_{\text {sun }}^{\mathrm{w}}\right)+\left(1 / \mu^{\mathrm{w}}\right)\right]
$$

is known $\forall \mathbf{x}^{\mathrm{s}}$, and any $\theta_{\mathrm{LOS}}^{\mathrm{a}}$. Using Eq. (33) in Eq. (31),

$$
\tilde{i}^{\mathrm{pol}}\left(\mathbf{x}^{\mathrm{s}}\right)=C-\beta^{\mathrm{w}} \tilde{z}\left(\mathbf{x}^{\mathrm{s}}\right) .
$$

Thus, a plot of the measured $\tilde{i}^{\mathrm{pol}}\left(\mathbf{x}^{\mathrm{s}}\right)$ as a function of the known values of $\tilde{z}\left(\mathbf{x}^{\mathrm{s}}\right)$ fits a line. According to Eq. (34), the (negative) slope of this line yields an estimate $\hat{\beta}^{\mathrm{w}}$ of the water attenuation coefficient.

\section{Discussion}

A flying multiangular set of cameras captures, essentially, a slice of the Earth's light field. The satellite in orbit forms a huge, planet-sized light field camera, which over time captures a 3D subspace of the 5D spatiotemporal light field. Interestingly, the light field concept has recently evolved [9] to treat short distance descattering problems using active illumination. In a sense, multiangular (light field) sensing performs a set of tomographic projections of a multilayered scene. Tomography is used to recover multilayered objects is many fields. Also in our case, multi angular imaging is the main force. Polarization and soundings are auxiliary sources of information that greatly simplify the needed parameter estimation. Thus, passive light-field polarization imaging [13] appears give a handle on multilayer $[6,7]$ scattering and refraction problems. Conducting experiments to test our theory will require systems under current development such as those in Refs. [5, 12].

Multispectral analysis can be assisted by the method. The spectral distributions $\tau^{\mathrm{atm}}(\lambda)$ and $\beta^{\mathrm{w}}(\lambda)$ are respectively sensitive to the aerosol and hydrosol types and distributions [22]. In the most simple form, parameter estimation can be done per wavelength channel $\lambda$. The recovered functions $\hat{\beta}^{\mathrm{w}}(\lambda)$ and $\hat{\tau}^{\mathrm{atm}}(\lambda)$ may then be used in aerosol and hydrosol retrieval $[3,26]$. Multispectral data analysis can also eliminate object specularities [37].

In some cases, our assumptions will not be good enough. Assumption 1 does not hold if the water or atmospheric characteristics change significantly within the FOV. Assumption 2 does not hold if the entire FOV has no point which is "very deep." A more sophisticated model would be needed to allow for gradual lateral changes of media and calibrating components without deep water.

\section{Acknowledgments}

We thank the reviewers for the useful comments. Yoav Schechner is a Landau Fellow - supported by the Taub Foundation. The research is supported in part by Department of the Navy Grant N62909-10-1-4056 issued by the Office of Naval Research Global. Part of this work was performed at the Jet Propulsion Laboratory, California Institute of Technology, under contract with NASA.

\section{References}

[1] D. E. Bowker, R. E. Davis, D. L. Myrick, K. Stacy and W. T. Jones, "Spectral reflectances of natural targets for use in remote sensing studies," NASA Ref. Pub. 1139, 1985. 5

[2] F. M. Bréon, "An analytical model for the cloud free atmosphere/ocean system reflectance," Remote Sens. Environ., vol. 43, pp. 179-192, 1993. 2, 3, 4, 6

[3] G. C. Chang and R. W. Gould Jr., "Comparisons of optical properties of the coastal ocean derived from satellite ocean color and in situ measurements," Opt. Exp. vol. 14, pp. 10149-10163, 2006. 1, 7 
[4] S. V. Cristina, P. Goela, J. D. Icely, A. Newton and B. Fragoso, "Assessment of water-leaving reflectances of oceanic and coastal waters using MERIS satellite products off the southwest coast of Portugal," J. Coast. Res. vol. 56, pp. 1479-1483, 2009. 1, 6

[5] D. J. Diner, A. Davis, B. Hancock, S. Geier, B. Sheingans, V. Jovanovic, M. Bull, D. M. Rider, R. A. Chipman, A.-B. Mahler and S. C. McClain, "First results from a dual photoelastic-modulator-based polarimetric camera," App. Opt., vol. 49, pp. 2929-2946, 2010. 2, 7

[6] C. Donner and H. Wann Jensen, "Light diffusion in multilayered translucent materials," ACM TOG 24(3), 2005. 7

[7] C. Donner, T. Weyrich, E. d'Eon, R. Ramamoorthi and S. Rusinkiewicz, "A layered, heterogeneous reflectance model for acquiring and rendering human skin," ACM TOG 27(5), 2008. 7

[8] R. Fattal, "Single image dehazing," ACM TOG 27, 2008. 3

[9] C. Fuchs, M. Heinz, M. Levoy, H.-P. Seidel, H. P. A. Lensch, "Combining confocal imaging and descattering," Eurographics Symposium on Rendering, 2008. 7

[10] H. R. Gordon, "Normalized water-leaving radiance: revisiting the influence of surface roughness," App. Opt., vol. 44, pp. 241-248, 2005. 4

[11] J. E. Hansen and L. D. Travis, "Light scattering in planetary atmospheres," Space Science Rev., 16:527-610, 1974. 2, 3

[12] B. A. Hooper, B. Baxter, C. Piotrowski, J. Z. Williams and J. Dugan "An airborne imaging multispectral polarimeter," Proc. IEEE/MTS Oceans 2009. 2, 7

[13] R. Horstmeyer, G. Euliss, R. Athale and M. Levoy "Flexible multimodal camera using a light field architecture," Proc. IEEE ICCP 2009. 7

[14] K. He, J. Sun, X. Tang, "Single image haze removal using dark channel prior," Proc. IEEE CVPR 2009. 4

[15] I. Ihrke, K. N. Kutulakos, H. P. A. Lensch, M. Magnor and W. Heidrich "State of the art in transparent and specular object reconstruction," Proc. Eurographics 2008. 3

[16] B. Jähne, J. Klinkle and S. Waas, "Imaging of short ocean wind waves: a critical theoretical review," JOSA A vol. 11, pp. 2197-2209, 1994. 3

[17] R. Kaftory, Y. Y. Schechner and J. Zeevi, "Variational distance dependent image restoration," Proc. IEEE CVPR, 2007. 5

[18] G. W. Kattawar and C. N. Adams, "Stokes vector calculations of the submarine light field in an atmosphere-ocean with scattering according to a Rayleigh phase matrix: effect of interface refractive index on radiance and polarization," Limnol. Oceanogr., 34:1453-1472, 1989. 2, 3, 7

[19] J. Kopf, B. Neubert, B. Chen, M. Cohen, D. Cohen-Or, O. Deussen, M. Uyttendaele and D. Lischinski, "Deep Photo: model-based photograph enhancement and viewing," ACM TOG, vol. 27, 2008. 4

[20] L. Kratz and K. Nishino, "Factorizing scene albedo and depth from a single foggy image," IEEE ICCV 2009. 4
[21] D. R. Lyzenga "Passive remote sensing techniques for mapping water depth and bottom features," App. Opt., vol. 17, pp. 379-383, 1978. 1, 2, 3, 4

[22] J. V. Martonchik, D. J. Diner, R. A. Kahn, T. P. Ackerman, M. M. Verstrate, B. Pinty and H. R. Gordon "Techniques for the retreival of aerosol properties over land and ocean using multiangle imaging," IEEE TGRS 36:1212-1227, 1998. 1, 2, $4,5,6,7$

[23] J. V. Martonchik, R. A. Kahn and D. J. Diner, "Retrieval of aerosol properties over land using MISR observations", in Satellite Remote Sensing Over Land, eds. A. A. Kokhanovsky and G. deLeeuw, pp. 267- 293, Springer Praxis, Berlin 2009. 5

[24] D. Miyazaki and K. Ikeuchi, "Polarization estimation of transparent objects by using inverse polarization ray tracing," IEEE Trans. PAMI, vol. 29, pp. 2018-2029, 2007. 2, 3

[25] C. D. Mobley, Light and Water, Radiative Transfer in Natural Waters, Ch. 11, Academic Press, Boston 1994. 3

[26] W. J. Moses, A. A. Gitelson, S. Berdnikov and V. Povazhnyy, "Estimation of cholorophyll- $a$ concentration in case II waters using MODIS and MERIS data - successes and challanges," Environ. Res. Lett. vol. 4, 2009. 1, 7

[27] S. G. Narasimhan and S. K. Nayar, "Vision and the atmosphere,” IJCV, vol. 48, pp. 233-254, 2002. 3, 4

[28] W. D. Philpot "Bathymetric mapping with passive multispectral imagery," App. Opt., vol. 28, pp. 1569-1578, 1989. 1, 4

[29] Y. Y. Schechner and N. Karpel, "Recovery of underwater visibility and structure by polarization analysis," IEEE JOE, vol. 30, pp. 570-587, 2005. 3, 4

[30] Y. Y. Schechner, S. G. Narasimhan, and S. K. Nayar, "Polarization-based vision through haze," App. Opt., vol. 42, pp. 511-525, 2003. 3, 4

[31] R. P. Stumpf and K. Holderied "Determination of water depth with high resolution satellite imagery over variable bottom types," Limnol. Oceangr., 48:547-556, 2003. 1, 3

[32] R. T. Tan, "Visibility in bad weather from a single image," Proc. IEEE CVPR 2008. 3, 4

[33] T. Treibitz and Y. Y. Schechner, "Recovery limits in pointwise degradation,” Proc. IEEE ICCP 2009. 5

[34] K. J. Voss and A. L. Chapin, "Upwelling radiance distribution camera system NURADS," Opt. Exp., vol. 13, pp. 42504262, 2005. 3

[35] K. J. Voss and A. Morel, "Bidirectional reflectance function for oceanic waters with varying chlorophyll concentrations: measurements versus predictions," Limnol. Oceanogr., vol. 50, pp. 698-705, 2005. 3

[36] H. Zhang and K. J. Voss, "Bidirectional reflectance study on dry, wet, and submerged particulate layers: effects of pore liquid refractive index and translucent particle concentrations," App. Opt., vol. 45, pp. 8753-8763, 2006. 3, 4, 5

[37] T. Zickler, S. P. Mallick, D. J. Kriegman and P. N. Belhumeur "Color subspaces as photometric invariants," IJCV, vol. 79, pp. 13-30, 2008. 7 